\title{
On the diameter of sets of almost powers
}

\author{
by \\ B. M. M. DE WEGER (Krimpen aan den IJssel) and \\ C. E. VAn DE WoestiJne (Leiden)
}

1. Introduction and main results. The well-known theory of the Pell equation states that for any squarefree $d \in \mathbb{Z}$, there are infinitely many pairs of integers $(x, y)$ such that $x^{2}-d y^{2}=1$. Furthermore, one observes that the consecutive integers 48, 49 and 50 are respectively 3,1 and 2 times a square. But these seem to be the only cases where two or more almost powers are so close to each other.

Our objective in this paper is to prove both upper and lower bounds on the size of intervals containing a number of $n$ almost powers $a_{i} x_{i}^{k}$ with the $a_{i}$ fixed, and to construct examples showing that these bounds are sharp in some sense.

For the related problem of estimating the size of the $a_{i}$ when we take our $n$ almost powers to be consecutive integers, we refer to [10].

Let $n, k \in \mathbb{N}, n \geq 3$ and $k \geq 2$. Let $a_{1}, \ldots, a_{n}$ be given positive integers (the "weights") such that $a_{1}^{-1 / k}, \ldots, a_{n}^{-1 / k}$ are $\mathbb{Z}$-linearly independent. We define the almost-power diameter $\operatorname{diam}(X)$ of $X \in \mathbb{N}$ as the least possible diameter of a set $S \subset \mathbb{N}$ containing both $X$ and, for $j=1, \ldots, n$, a "weighted power" $a_{j} x_{j}^{k}$ (here we define the diameter of a finite subset $S \subset \mathbb{R}$ as $\left.\max _{s, t \in S}|s-t|\right)$. It is clear that if any such set indeed has a minimal diameter, each of its elements will be either $a_{j} x_{j}^{* k}$ or $a_{j}\left(x_{j}^{*}+1\right)^{k}$, where $x_{j}^{*}=\left\lfloor\sqrt[k]{X / a_{j}}\right\rfloor$.

For example, if $k=2, n=3, a_{1}=3, a_{2}=2, a_{3}=1$, then $\operatorname{diam}(48)=$ $\operatorname{diam}(49)=\operatorname{diam}(50)=2$, corresponding to the above mentioned triple 48 , 49, 50. From now on, we will abbreviate our term to just "diameter".

We want to know how small and how large diam $(X)$ can be compared to $X$. In Section 2 we prove the upper bound declared below. Let us introduce the following number, depending on $n, k$, and the weights $a_{i}$ :

1991 Mathematics Subject Classification: Primary 11N25; Secondary 11A05, 11Y70. 


$$
\vartheta=k \max _{I \subset\{1, \ldots, n\}} \frac{1}{|I|+1} \sum_{j \in I} a_{j}^{1 / k} .
$$

We will assume in the sequel that $a_{1}>\ldots>a_{n}$. Under this assumption, we can write

$$
\vartheta=k \max _{1 \leq i \leq n} \frac{1}{i+1} \sum_{j=1}^{i} a_{j}^{1 / k} .
$$

For example, if $k=2, n=3, a_{1}=3, a_{2}=2, a_{3}=1$, then $\vartheta=$ $\frac{2}{3}(\sqrt{3}+\sqrt{2})=2.0975 \ldots$ The following theorem states that $\vartheta X^{1-1 / k}$ is essentially the best uniform upper bound for $\operatorname{diam}(X)$.

TheOrem 1.1. For given $n, k, a_{1}, \ldots, a_{n}$ as defined above, we have

$$
\limsup _{X \rightarrow \infty} \frac{\operatorname{diam}(X)}{X^{1-1 / k}}=\vartheta .
$$

In Section 3, we derive several lower bounds for diam $(X)$. By making use of Schmidt's generalization of the Thue-Siegel-Roth theorem, we prove

TheORem 1.2. For every $\varepsilon>0$ there exists a constant $c$, depending only on $\max _{1 \leq i \leq n} a_{i}$ and $\varepsilon$, such that for all $X \in \mathbb{N}$ we have

$$
\operatorname{diam}(X)>c X^{1-1 / k-1 /(k(n-1))-\varepsilon} .
$$

As to the sharpness of this bound, we have

THEOREM 1.3. There exists a positive constant $\psi=\psi\left(n, k, a_{1}, \ldots, a_{n}\right)$ such that, for every $\varepsilon>0$, there are infinitely many $X \in \mathbb{N}$ with

$$
\operatorname{diam}(X)<(\psi+\varepsilon) X^{1-1 / k-1 /(k(n-1))} .
$$

An explicit value for $\psi$ follows from the proof.

We explore two ways of providing effective lower bounds for $\operatorname{diam}(X)$. If $k$ is very large, then we can successfully apply the theory of linear forms in logarithms, and obtain an effective lower bound for $\operatorname{diam}(X)$. Indeed, we prove that there exists a (very large) effectively computable constant $C$, depending only on $\max _{1 \leq i \leq n} a_{i}$, such that for all $X \in \mathbb{N}$ we have

$$
\operatorname{diam}(X)>X^{1-C \log k / k},
$$

a result which is nontrivial only when $k \gg C \log C$.

Furthermore, we apply effective simultaneous irrationality measures to obtain lower bounds on $\operatorname{diam}(X)$ for specific sets of $a_{i}$ 's. As an example, we show that, if $n=3, k=2, a_{1}=6, a_{2}=3$, and $a_{3}=2$, we have

$$
\operatorname{diam}(X)>X^{1-1 / k-0.79155 / k}=X^{0.10423}
$$

for $X$ sufficiently large. 
The proof of Theorem 1.2 is a relatively straightforward application of Schmidt's theorem. However, the proof of Theorem 1.1, besides using an extension of Kronecker's Theorem on simultaneous inhomogeneous Diophantine approximations, achieves a lot with elementary means and gives a lot of information, whereas Theorem 1.3 is a simple application of Dirichlet's Theorem. This means that recent techniques of computational Diophantine approximation theory can be used for producing explicit examples of $X$ for which the inequalities for $\operatorname{diam}(X)$ of Theorems 1.1 and 1.3 are true with a given accuracy. In Section 4 we list some results obtained with this method.

There is yet another technique, laborious however, which makes it possible to determine effectively all $X \in \mathbb{N}$ with $\operatorname{diam}(X) \leq \Delta_{0}$ for a given $\Delta_{0}$ which is not too large. Namely, one considers for each $\Delta \in \mathbb{Z}$ with $|\Delta| \leq \Delta_{0}$ the equations $a_{j} x_{j}^{k}-a_{i} x_{i}^{k}=\Delta$ for some fixed $i \in\{1, \ldots, n\}$ and for all $j \in\{1, \ldots, n\} \backslash\{i\}$. If $k \geq 3$ then each such equation is a Thue equation, that can be solved by standard, but laborious, methods (cf. [8]). If $k=2$ we consider two such equations for different $i$ 's, and we have a system of simultaneous Pell equations, which can also be solved by standard methods (cf. [2]).

Acknowledgements. We would like to thank R. Tijdeman and the referee for their many valuable comments, and F. Göbel for suggesting the problem.

2. A tight upper bound for $\operatorname{diam}(X)$. In this section, we will prove two propositions which together yield Theorem 1.1. We assume that we are given positive integers $n \geq 2, k \geq 2$, and $a_{1}>\ldots>a_{n}$ such that the numbers $a_{i}^{-1 / k}, i=1, \ldots, n$, are linearly independent over $\mathbb{Z}$. Furthermore, let $X$ be a positive integer, and $x_{j}^{*}=\left\lfloor\sqrt[k]{X / a_{j}}\right\rfloor$, as designated in the introduction.

2.1. Notation and results. First, for $I \subset\{1, \ldots, n\}$ we define

$$
\sigma_{I}=\frac{k}{|I|+1} \sum_{j \in I} a_{j}^{1 / k}
$$

Thus, we may write that $\vartheta$ is defined as

$$
\vartheta=\max _{I \subset\{1, \ldots, n\}} \sigma_{I} \text {. }
$$

Next, let $\boldsymbol{i}=\left(i_{1}, \ldots, i_{n}\right), \boldsymbol{j}=\left(j_{1}, \ldots, j_{n}\right)$ be permutations of $(1, \ldots, n)$. We put $i_{n+1}=j_{1}$ and $j_{0}=i_{n}$, and go on to consider the sets $\gamma_{l, r}$, defined by

$$
\gamma_{l, r}=\left\{a_{i_{l}} x_{i_{l}}^{* k}, \ldots, a_{i_{n}} x_{i_{n}}^{* k}, a_{j_{1}}\left(x_{j_{1}}^{*}+1\right)^{k}, \ldots, a_{j_{r}}\left(x_{j_{r}}^{*}+1\right)^{k}\right\}
$$

for $1 \leq l \leq n+1$, and $0 \leq r \leq n$. A subset $\gamma_{l, r}$ will be called minimal if $\left\{i_{l}, \ldots, i_{n}, j_{1}, \ldots, j_{r}\right\} \supset\{1, \ldots, n\}$, and this property is not shared by $\gamma_{l+1, r}$ and $\gamma_{l, r-1}$. We denote the $l$ and $r$ indices of all minimal subsets, in 
increasing order, by $l_{0}=1, l_{1}, \ldots, l_{m}=n+1$ and $r_{0}=0, r_{1}, \ldots, r_{m}=n$ $\left(\gamma_{1,0}\right.$ and $\gamma_{n+1, n}$ are clearly minimal in all cases $)$ and thus find $1 \leq m \leq n$.

LEMMA 2.1. In the above notation, we have $i_{l_{s}}=j_{r_{s+1}}$ for $s=0, \ldots, m-1$.

Pro of. By definition, $\gamma_{l_{s}, r_{s}}$ is minimal, hence the set $\left\{i_{l_{s}+1}, i_{l_{s}+2}, \ldots, i_{n}\right.$, $\left.j_{1}, \ldots, j_{r_{s}}\right\}$ does not contain $i_{l_{s}}$. By subsequently adjoining $j_{r_{s}+1}, j_{r_{s}+2}, \ldots$ to this set, we eventually obtain a set that contains $i_{l_{s}}$, and thus contains a minimal subset, which must be $\gamma_{l_{s+1}, r_{s+1}}$ by definition. As $j_{r_{s+1}}$ was the last adjoined element, we conclude that $j_{r_{s+1}}=i_{l_{s}}$.

We have obtained the following results. The first one implies the validity of the given upper bound, whereas the second one proves its tightness.

Proposition 2.2. Let $X$ be a positive integer, and $m$ and $x_{j}^{*}$ as defined above. Take the permutations $\boldsymbol{i}$ and $\boldsymbol{j}$ such that

$$
a_{i_{1}} x_{i_{1}}^{* k}<\ldots<a_{i_{n}} x_{i_{n}}^{* k} \leq X<a_{j_{1}}\left(x_{j_{1}}^{*}+1\right)^{k}<\ldots<a_{j_{n}}\left(x_{j_{n}}^{*}+1\right)^{k} .
$$

Then

$$
\operatorname{diam}(X) \leq \frac{k}{m+1} \sum_{s=1}^{m} a_{j_{r_{s}}}^{1 / k} X^{1-1 / k}+O\left(X^{1-2 / k}\right) .
$$

Proposition 2.3. Let $T=\left\{t_{1}, \ldots, t_{v}\right\} \subset\{1, \ldots, n\}$ be such that $\sigma_{T}<$ $k a_{t_{s}}^{1 / k}$ for $s=1, \ldots, v$. Then for all $\varepsilon>0$, there are infinitely many positive integers $X$ which satisfy

$$
\begin{gathered}
\operatorname{diam}(X)=\sigma_{T} X^{1-1 / k}+O\left(X^{1-1 / k-(1-\varepsilon) /(v k)}\right), \\
a_{t_{1}} x_{t_{1}}^{* k}<\ldots<a_{t_{v}} x_{t_{v}}^{* k} \leq X<a_{t_{1}}\left(x_{t_{1}}^{*}+1\right)^{k}<\ldots<a_{t_{v}}\left(x_{t_{v}}^{*}+1\right)^{k},
\end{gathered}
$$

using the notations of Proposition 2.2.

From these propositions, we have

Corollary 2.4. (i) For every $\varepsilon>0$ there are only finitely many $X \in \mathbb{N}$ with $\operatorname{diam}(X)>(\vartheta+\varepsilon) X^{1-1 / k}$.

(ii) For every $\varepsilon>0$ there are infinitely many $X \in \mathbb{N}$ with $\operatorname{diam}(X)>$ $(\vartheta-\varepsilon) X^{1-1 / k}$.

In the proof of this corollary, which clearly implies Theorem 1.1, we make use of the following lemma, which provides an alternative definition for $\vartheta$.

LEMma 2.5. The maximality condition imposed on subsets $I$ of $\{1, \ldots, n\}$ in (2) is equivalent to the condition that

$$
k a_{i}^{1 / k} \leq \sigma_{I} \leq k a_{j}^{1 / k} \quad \text { for all } i \in\{1, \ldots, n\} \backslash I \text { and all } j \in I .
$$

Proof. Let $I \subset\{1, \ldots, n\}$; write $m=|I|$. Let $I^{\prime}$ be obtained by leaving $l$ elements out of $I$, say $s_{1}, \ldots, s_{l}$, and adding $v$ elements, say $t_{1}, \ldots, t_{v}$. 
Then

(6)

$$
\begin{aligned}
\sigma_{I} & \geq \sigma_{I^{\prime}} \\
& \Leftrightarrow\left(1-\frac{m+1}{m-l+v+1}\right) \sigma_{I} \geq \frac{k}{m-l+v+1}\left(\sum_{j=1}^{v} a_{t_{j}}^{1 / k}-\sum_{j=1}^{l} a_{s_{j}}^{1 / k}\right) \\
& \Leftrightarrow(v-l) \sigma_{I} \geq k\left(\sum_{j=1}^{v} a_{t_{j}}^{1 / k}-\sum_{j=1}^{l} a_{s_{j}}^{1 / k}\right) .
\end{aligned}
$$

First assume that $\sigma_{I} \geq \sigma_{I^{\prime}}$ for all $I^{\prime} \subset\{1, \ldots, n\}$. Then taking first $l=0$ and $v=1$ and then $l=1$ and $v=0$, we see that (5) is satisfied.

Next assume that we have (5); note that this implies the right hand side of (6). Then the above considerations show that $\sigma_{I}$ is indeed maximal.

Proof of Corollary 2.4. Let $I$ be that subset of $\{1, \ldots, n\}$ for which $\sigma_{I}$ is maximized, and thus equal to $\vartheta$. The first claim follows from Proposition 2.2 by considering the case where $\left\{j_{r_{s}} \mid s=1, \ldots, m\right\}=I$. As to the second one, our lemma asserts that $T=I$ satisfies the conditions of Proposition 2.3 , so that our claim follows from the definition of $\vartheta$.

Unfortunately, (4b) is not enough to prove that $T=\left\{j_{r_{s}} \mid s=1, \ldots, m\right\}$, which would constitute equivalence between maximality of $\operatorname{diam}(X)$ and the condition that $\frac{k}{m+1} \sum_{s=1}^{m} a_{j_{r_{s}}}^{1 / k}$ be equal to $\vartheta$. However, with the aid of Lemma 2.1, it is easy to verify that $T$ does have this property if there is a $u, 1 \leq u \leq n$, such that $i_{1}=t_{1}, \ldots, i_{u}=t_{u}, j_{n-(v-u)}=t_{u}, \ldots, j_{n}=t_{v}$-in other words, if the terms in (3) with indices from $T$ are either less or greater than all other terms. Numerical experiments by the authors show that the majority of extremal diameter examples indeed have this property, whereas a minority do not.

2.2. Proof of Proposition 2.2. We make use of a less informative version of Proposition 2.2, which follows at once from the observation that $x^{k}$ and $(x+1)^{k}$ differ by $\mathcal{O}\left(x^{k-1}\right)$.

LEMmA 2.6. There exists a real number $\varphi$, depending on $k$ and $\max _{i} a_{i}$, such that for all $X \in \mathbb{N}$,

$$
\operatorname{diam}(X)<\varphi X^{1-1 / k}
$$

Proof. By the definition of $x_{j}^{*}$ we have

$$
\left|X-a_{j} x_{j}^{k}\right| \leq a_{j}\left(\left(x_{j}^{*}+1\right)^{k}-x_{j}^{* k}\right) \leq a_{j}\left(k x_{j}^{* k-1}+\ldots+1\right) \leq \varphi_{1} x_{j}^{* k-1}
$$

for some constant $\varphi_{1}$, and thus for any $i$ and $j$ we have

$$
\left|a_{i} x_{i}^{k}-a_{j} x_{j}^{k}\right| \leq\left|a_{i} x_{i}^{k}-X\right|+\left|X-a_{j} x_{j}^{k}\right| \leq 2 \varphi_{1} x_{j}^{* k-1} \leq \varphi X^{1-1 / k}
$$

for some constant $\varphi$, which proves the lemma. 
We introduce one more notation. For $j=1, \ldots, n$, we define the numbers $\alpha_{j}$ by

$$
X-a_{j} x_{j}^{* k}=\alpha_{j} X^{1-1 / k} .
$$

Note that $\alpha_{j} \geq 0$ and, by Lemma 2.6, $\alpha_{j}=O(1)$ as $X \rightarrow \infty$.

Proof of Proposition 2.2. Note that $x_{j}^{*} \leq a_{j}^{-1 / k} X^{1 / k}<x_{j}^{*}+1$ for any $j \in\{1, \ldots, n\}$, so that

$$
x_{j}^{*}=\left(X / a_{j}\right)^{1 / k}+O(1) .
$$

Together with (7), this implies

$$
\begin{aligned}
a_{j}\left(x_{j}^{*}+1\right)^{k}-X & =a_{j} x_{j}^{* k}+k a_{j} x_{j}^{* k-1}+O\left(x_{j}^{* k-2}\right)-X \\
& =k a_{j} x_{j}^{* k-1}-\alpha_{j} X^{1-1 / k}+O\left(x_{j}^{* k-2}\right) \\
& =\left(k a_{j}^{1 / k}-\alpha_{j}\right) X^{1-1 / k}+O\left(X^{1-2 / k}\right) .
\end{aligned}
$$

Put

$$
\begin{aligned}
& \beta_{0}=X-a_{i_{1}} x_{i_{1}}^{* k}, \\
& \beta_{s}=a_{j_{r_{s}}}\left(x_{j_{r_{s}}}^{*}+1\right)^{k}-a_{i_{l_{s}}} x_{i_{l_{s}}}^{* k} \quad \text { for } s=1, \ldots, m-1, \\
& \beta_{m}=a_{j_{n}}\left(x_{j_{n}}^{*}+1\right)^{k}-X .
\end{aligned}
$$

Then by definition

$$
\operatorname{diam}(X)=\min _{0 \leq s \leq m} \beta_{s} .
$$

Using (7) and Lemma 2.1, we find

$$
\begin{aligned}
\beta_{0} & =\alpha_{i_{l_{0}}} X^{1-1 / k}=\alpha_{{j_{r}}_{1}} X^{1-1 / k} \\
\beta_{s} & =\left(k a_{j_{r_{s}}}^{1 / k}-\alpha_{{j_{r}}_{s}}+\alpha_{i_{l_{s}}}\right) X^{1-1 / k}+O\left(X^{1-2 / k}\right) \\
& =\left(k a_{j_{r_{s}}}^{1 / k}-\alpha_{{j_{r_{s}}}_{1}}+\alpha_{{j_{r_{s+1}}}}\right) X^{1-1 / k}+O\left(X^{1-2 / k}\right) \quad \text { for } s=1, \ldots, m-1, \\
\beta_{m} & =\left(k a_{j_{r_{m}}}^{1 / k}-\alpha_{j_{r_{m}}}\right) X^{1-1 / k}+O\left(X^{1-2 / k}\right) .
\end{aligned}
$$

Notice that in the sum of the $\beta_{s}$ the $\alpha$ 's cancel out. As the minimum of the $\beta_{s}$ 's cannot exceed the average, we have

$$
\operatorname{diam}(X) \leq \frac{1}{m+1} \sum_{s=0}^{m} \beta_{s}=\frac{k}{m+1} \sum_{s=1}^{m} a_{j_{r_{s}}}^{1 / k} X^{1-1 / k}+O\left(X^{1-2 / k}\right)
$$

2.3. Proof of Proposition 2.3. We need a theorem of M. Sweet [6] about the number of solutions of certain systems of Diophantine inequalities, which we state as a lemma, in a somewhat simplified version which fits our purposes (e.g., we take $s=1$ in Sweet's notation).

Lemma 2.7. Let $\eta_{s}, \theta_{s} \in \mathbb{R}$ for $s=1, \ldots, v, v \geq 1$. Suppose $\psi$ is a decreasing function on the positive reals with values between 0 and 1 , and 
assume that $\int_{1}^{\infty} \psi(t)^{v} d t$ diverges. Let $\lambda(k)$ denote the number of solutions $q \in \mathbb{Z}, \vec{p}=\left(p_{1}, \ldots, p_{v}\right) \in \mathbb{Z}^{v}$ of the system

$$
0 \leq q \eta_{s}-p_{s}-\theta_{s}<\psi(q), \quad s=1, \ldots, v,
$$

with $1 \leq q \leq k$. Let $g$ be a positive increasing (real) function such that

$$
\left|\sum_{s=1}^{v} \eta_{s} p_{s}-q\right| \geq \frac{1}{P^{v} g(P)}
$$

for all $q \in \mathbb{Z}$ and $\vec{p} \in \mathbb{Z}^{v}$, with $P=\max \left|p_{i}\right|$ sufficiently large (thus the matrix $\left[\eta_{1}, \ldots, \eta_{v}\right]^{T}$ has type $\leq g$ in Sweet's terminology). If

$$
F(t)^{v+2}:=\psi(t)^{v} \operatorname{tg}\left(t^{1 / v}\right)^{-1}
$$

is increasing to infinity, we have

$$
\lambda(k)=2 \int_{1}^{k} \psi(t)^{v} d t+O\left(\int_{1}^{k} \psi(t)^{v} F(t)^{-1} d t\right) .
$$

Proof of Proposition 2.3. As may be seen from the proof of Proposition $2.2, \operatorname{diam}(X)$ is maximized if all $\beta$ 's are equal. Therefore we study the linear system

$$
\gamma_{1}=k a_{t_{s}}^{1 / k}-\gamma_{s}+\gamma_{s+1}=k a_{t_{v}}^{1 / k}-\gamma_{v} \quad \text { for } s=1, \ldots, v-1,
$$

which is obtained from the system $\beta_{0}=\beta_{1}=\ldots=\beta_{m}$ by leaving the lower order terms out. Note that this system has a unique solution, which has $\gamma_{1}$ equal to $\sigma_{T}$.

Now our aim is to find $x_{t_{s}}^{*} \in \mathbb{Z}$ such that the corresponding $\alpha_{t_{s}}$ are good approximations of the $\gamma_{s}$. Let $b$ be such that $\left\{b^{-1 / k}\right\} \cup\left\{a_{t_{s}}^{-1 / k} \mid s=1, \ldots, v\right\}$ is a $\mathbb{Z}$-linear independent set; in most cases, $b=1$ will suffice, or $b=2 / 3$ if $a_{t_{s}}=1$ for some $s$ already. By the theorem of Kronecker on inhomogeneous simultaneous Diophantine approximation, there are positive integers $y$ and $x_{t_{s}}^{*}$ (for $s=1, \ldots, v$ ) such that, for all positive $\varepsilon$, we have

$$
\left|\left(\frac{b}{a_{t_{s}}}\right)^{1 / k} y-x_{t_{s}}^{*}-\frac{\gamma_{s}}{k a_{t_{s}}^{1 / k}}\right|<y^{(-1+\varepsilon) / v} .
$$

In fact, we can apply Lemma 2.7 to show that there are infinitely many solutions to this system.

Set $\psi(t)=t^{(-1+\varepsilon) / v}$; it is clear that $\lim _{T \rightarrow \infty} \int_{1}^{T} t^{-1+\varepsilon} d t=\infty$. Take further $\eta_{s}=\left(b / a_{t_{s}}\right)^{1 / k}$, and $\theta_{s}=\gamma_{s} /\left(k a_{t_{s}}^{1 / k}\right)(s=1, \ldots, v)$. In order to verify the assertion about $g$, we apply a generalization of Roth's Theorem by Schmidt [5, Corollary VI.1E] to find that for any $\delta>0$ there is a positive 
constant $c_{\delta}$ such that

$$
\left|\sum_{s=1}^{v} \eta_{s} p_{s}-q\right| \geq c_{\delta} P^{-v-\delta}
$$

for all $q \in \mathbb{Z}$ and $\vec{p}=\left(p_{1}, \ldots, p_{v}\right) \in \mathbb{Z}^{v}$, where $P=\max \left|p_{i}\right|>0$. Choose $\delta$ such that $0<\delta<v \varepsilon$ and take $g(t)=t^{\delta} / c_{\delta}$. Then

$$
F(t)^{v+2}=\psi(t)^{v} \operatorname{tg}\left(t^{1 / v}\right)^{-1}=c_{\delta} t^{\varepsilon-\delta / v}
$$

increases to infinity with $t$. As all conditions are satisfied, Lemma 2.7 allows us to conclude that the number of solutions $\lambda(\kappa)$ of (10) with $y<\kappa$ satisfies

$$
\lambda(\kappa)=2 \int_{1}^{\kappa} t^{-1+\varepsilon} d t+O\left(\int_{1}^{\kappa} t^{-1+\varepsilon} F(t)^{-1} d t\right)
$$

which clearly grows to infinity as $\kappa$ does. have

Let $\left(y, x_{t_{1}}^{*}, \ldots, x_{t_{v}}^{*}\right) \in \mathbb{Z}^{v+1}$ be a solution to (10); put $X=b y^{k}$. Now we

$$
\begin{aligned}
\alpha_{t_{s}} X^{1-1 / k} & =X-a_{t_{s}} x_{t_{s}}^{* k} \\
& =b y^{k}-a_{t_{s}}\left(\left(\frac{b}{a_{t_{s}}}\right)^{1 / k} y-\frac{\gamma_{s}}{k a_{t_{s}}^{1 / k}}+O\left(y^{(-1+\varepsilon) / v}\right)\right)^{k} \\
& =\gamma_{s} b^{1-1 / k} y^{k-1}+O\left(y^{k-1-(1-\varepsilon) / v}\right) \\
& =\gamma_{s} X^{1-1 / k}+O\left(X^{1-1 / k-(1-\varepsilon) /(v k)}\right)
\end{aligned}
$$

so that $\alpha_{t_{s}}=\gamma_{s}+O\left(X^{(-1+\varepsilon) /(v k)}\right)$, as required.

We claim that there exists a constant $c>0$ such that $\gamma_{v} \geq c$, and $\gamma_{s}-\gamma_{s+1} \geq c$ for $s=1, \ldots, v-1$. To see this, note first that $\gamma_{s}=\gamma_{s+1}$ for an $s$, resp. $\gamma_{v}=0$, is impossible because of the $\mathbb{Z}$-linear independence of the $a_{i}^{-1 / k}$. Furthermore, if one such number is negative, we have a $t_{0} \in T$ with

$$
k a_{t_{0}}^{1 / k}<\gamma_{1}
$$

by (9), contradicting the assumptions of the proposition. Our claim follows.

We substitute our $c$ in (9); as we may assume that $X$ is sufficiently large, this gives us the existence of a constant $c^{\prime}>0$ with

$$
\alpha_{t_{s}}-\alpha_{t_{s+1}}>c^{\prime}, \quad \alpha_{t_{v}}>c^{\prime}
$$

This implies

$$
a_{t_{1}} x_{t_{1}}^{* k}<\ldots<a_{t_{v}} x_{t_{v}}^{* k}<X .
$$

Using (8), we also find

$$
X<a_{t_{1}}\left(x_{t_{1}}^{*}+1\right)^{k}<\ldots<a_{t_{v}}\left(x_{t_{v}}^{*}+1\right)^{k} .
$$


It is now easy to prove the correct lower bound for the diameter: by (9) and (12), we have

$$
\begin{aligned}
\beta_{s} & =\left(k a_{t_{s}}^{1 / k}-\alpha_{t_{s}}+\alpha_{t_{s+1}}\right) X^{1-1 / k}+O\left(X^{1-2 / k}\right) \\
& =\left(k a_{t_{s}}^{1 / k}-\gamma_{s}+\gamma_{s+1}\right) X^{1-1 / k}+O\left(X^{1-1 / k-(1-\varepsilon) /(v k)}\right) \\
& =\gamma_{1} X^{1-1 / k}+O\left(X^{1-1 / k-(1-\varepsilon) /(v k)}\right),
\end{aligned}
$$

for $s=1, \ldots, v-1$, whereas the same estimate for $\beta_{0}$ and $\beta_{v}$ may be derived analogously. Hence we find

$$
\operatorname{diam}(X)=\min _{0 \leq s \leq v} \beta_{s}=\sigma_{T} X^{1-1 / k}+O\left(X^{1-1 / k-(1-\varepsilon) /(v k)}\right)
$$

\section{Lower bounds for $\operatorname{diam}(X)$}

3.1. Proofs of Theorems 1.2 and 1.3. We start with Theorem 1.2. J. Turk has proved essentially the same result [7, Theorem 1]. However, as he uses a different definition of diameter, the details of his proof differ from ours.

Proof of Theorem 1.2. Let $\varepsilon>0$, and consider $X \in \mathbb{N}$ such that $\operatorname{diam}(X)$ $<X^{1-1 / k-1 /(k(n-1))-\varepsilon}$.

First let us restrict ourselves to $X$ of the form $a_{i_{0}} x^{k}$ for some $x \in \mathbb{N}$ and some $i_{0} \in\{1, \ldots, n\}$. For each $i \in\{1, \ldots, n\} \backslash\left\{i_{0}\right\}$ let $x_{i}$ be the integer such that $\left|a_{i_{0}} x^{k}-a_{i} x_{i}^{k}\right|$ is minimal. Then clearly there are constants $c, c^{\prime}$ such that for all $i$ we have

$$
\begin{aligned}
\left|x_{i}-\left(\frac{a_{i_{0}}}{a_{i}}\right)^{1 / k} x\right| & <c\left|a_{i_{0}} x^{k}-a_{i} x_{i}^{k}\right| x^{-(k-1)}<c \operatorname{diam}(X) x^{-(k-1)} \\
& <c^{\prime} x^{-1 /(n-1)-\varepsilon}<x^{-1 /(n-1)-\varepsilon / 2}
\end{aligned}
$$

if $x$ is large enough. Now Schmidt's generalization of the Thue-Siegel-Roth Theorem [5, Corollary VI.1C] tells us that there are only finitely many such $x$. The result follows.

Next consider general $X$. Define the integers $x_{i}^{*}(i=1, \ldots, n)$ and the indices $i_{1}, \ldots, i_{n}$ and $j_{1}, \ldots, j_{n}$ as in Proposition 2.2. Write $\varphi_{l}=a_{i_{l}} x_{i_{l}}^{* k}$ and $\psi_{r}=a_{j_{r}}\left(x_{j_{r}}^{*}+1\right)^{k}$ for $l, r=1, \ldots, n$. There are three cases:

(i) If $\operatorname{diam}(X)=\psi_{n}-X$, then

$\operatorname{diam}(X) \geq \operatorname{diam}\left(\psi_{n}\right)>\psi_{n}^{1-1 / k-1 /(k(n-1))-\varepsilon}>X^{1-1 / k-1 /(k(n-1))-\varepsilon}$.

(ii) If $\operatorname{diam}(X)=\psi_{r}-\varphi_{l}$ for some $l$ and $r$, then $\operatorname{diam}(X)=\operatorname{diam}\left(\varphi_{n}\right)$, i.e. of the form discussed above.

(iii) If $\operatorname{diam}(X)=X-\varphi_{1}$, then note that we have already shown that $\left|\varphi_{n}-\varphi_{1}\right|=\operatorname{diam}\left(\varphi_{n}\right)>\varphi_{n}^{1-1 / k-1 /(k(n-1))-\varepsilon}$, so that

$$
\begin{aligned}
\operatorname{diam}(X) & =\left(X-\varphi_{n}\right)+\left(\varphi_{n}-\varphi_{1}\right) \\
& >X-\varphi_{n}+\varphi_{n}^{1-1 / k-1 /(k(n-1))-\varepsilon}>X^{1-1 / k-1 /(k(n-1))-\varepsilon} .
\end{aligned}
$$


Here we use the inequality $B-A>B^{\alpha}-A^{\alpha}$, equivalent to $B-B^{\alpha}>A-A^{\alpha}$, which holds whenever $B>A>1$ and $0<\alpha<1$.

Hence we see that $X$ of general form with very small diameter can only occur in the (finite) neighbourhood of special $X$ with even smaller diameter. This proves the theorem.

We proceed with the proof of Theorem 1.3.

Proof of Theorem 1.3. We may assume that $a_{1}>\ldots>a_{n}$. Put

$$
\psi=k a_{n}^{1 /(k(n-1))}\left(a_{1}^{1 / k}+a_{2}^{1 / k}\right) .
$$

The theorem of Dirichlet on homogeneous simultaneous Diophantine approximation [5, Corollary II.1C] guarantees the existence of infinitely many solutions $\left(x_{1}, \ldots, x_{n}\right)$ in $\mathbb{Z}^{n}$ of the inequalities

$$
\left|x_{i}-\left(\frac{a_{n}}{a_{i}}\right)^{1 / k} x_{n}\right|<\frac{1}{x_{n}^{1 /(n-1)}} \quad(i=1, \ldots, n-1) .
$$

Let $\left(x_{1}, \ldots, x_{n}\right)$ be any such solution; then

$$
\begin{aligned}
\left|a_{n} x_{n}^{k}-a_{j} x_{j}^{k}\right|= & \left|a_{n}^{1 / k} x_{n}-a_{j}^{1 / k} x_{j}\right| \\
& \times\left|a_{n}^{1-1 / k} x_{n}^{k-1}+a_{n}^{1-2 / k} a_{j}^{1 / k} x_{n}^{k-2} x_{j}+\ldots+a_{j}^{1-1 / k} x_{j}^{k-1}\right| \\
< & a_{j}^{1 / k} x_{n}^{-1 /(n-1)}\left(k a_{n}^{1-1 / k} x_{n}^{k-1}+O\left(x_{n}^{k-2}\right)\right),
\end{aligned}
$$

and hence

$$
\begin{aligned}
\left|a_{i} x_{i}^{k}-a_{j} x_{j}^{k}\right|< & k a_{n}^{1 /(k(n-1))}\left(a_{i}^{1 / k}+a_{j}^{1 / k}\right)\left(a_{n} x_{n}^{k}\right)^{1-1 / k-1 /(k(n-1))} \\
& +O\left(x_{n}^{k-2-1 /(n-1)}\right) \\
< & (\psi+\varepsilon) X^{1-1 / k-1 /(k(n-1))},
\end{aligned}
$$

upon choosing $X=a_{n} x_{n}^{k}$, as we have done before.

For our standard example $k=2, n=3, a_{1}=3, a_{2}=2, a_{3}=1$, we find $\psi=2 \sqrt[4]{1}(\sqrt{3}+\sqrt{2})=6.293 \ldots$

3.2. Ways to obtain effective lower bounds. The ineffective nature of Schmidt's work called upon in the previous section gives no clue as to the existence or value of

$$
\liminf _{X \rightarrow \infty} \frac{\operatorname{diam}(X)}{X^{1-1 / k-1 /(k(n-1))}} .
$$

In this section, we explore two approaches that one may take to obtain explicit lower bounds to $\operatorname{diam}(X)$.

3.2.1. Linear forms in logarithms. The first approach is to apply the theory of linear forms in logarithms of algebraic numbers, as developed by 
Baker [1]. This results in the following theorem, which is nontrivial only when $k \gg C \log C$.

THeOREM 3.1. Let $k \geq 3$. There exists a (very large) effectively computable constant $C$, depending only on $a_{n-1}$ and $a_{n}$, such that for all $X \in \mathbb{N}$ we have

$$
\operatorname{diam}(X)>X^{1-C \log k / k} .
$$

Proof. This is an easy consequence of equation (1) of Proposition 1 in [7]: just take $m=k$ and $n=2$, and take the interval $[N, N+K]$ equal to the smallest set around $X$ containing all required almost powers, so that $K=\operatorname{diam}(X)$ (recall our definition of diameter). The result follows immediately.

3.2.2. Effective irrationality measures. The second approach makes use of results concerning (possibly simultaneous) effective irrationality measures for certain sets of algebraic numbers. The most recent results along these lines that we have found, have been obtained by Bennett [3]; for other authors, we refer to his bibliography. For a survey of the methods used in the proof of such results, see [4]. In the cited paper, Bennett shows, for instance, that

$$
\max \left\{\left|\sqrt{2}-p_{1} / q\right|,\left|\sqrt{3}-p_{2} / q\right|\right\}>q^{-1.79155}
$$

for all $p_{1}, p_{2}, q \in \mathbb{Z}$ with $q>q_{0}$ for an explicit $q_{0}$, or for all $q \in \mathbb{Z}$ if we allow for a somewhat lower exponent. The number $\lambda=1.79155$ is a simultaneous irrationality measure for $\{\sqrt{2}, \sqrt{3}\}$.

When comparing (14) to the Dirichlet approximation in the proof of Theorem 1.3, viz. (13), it is clear that we cannot apply this result directly to our problem, except when $n=2$ : instead of common denominators, the numbers whose $k$ th roots appear in the approximation system should have common numerators. However, consider finding $X$ of small diameter in the following setting: $n=3, k=2, a_{1}=6, a_{2}=3, a_{3}=2$. If we choose $x_{1}$ instead of $x_{3}$ as the "anchor variable" in (13), it follows that we try to find $x_{i} \in \mathbb{Z}$ such that

$$
\left|x_{2}-\sqrt{2} x_{1}\right| \text { and }\left|x_{3}-\sqrt{3} x_{1}\right|
$$

are simultaneously small. Now Bennett's result shows that at least one of these expressions must be greater than $x_{1}^{-0.79155}$ if $x_{1}$ is sufficiently large. Analogously to the proof of Theorem 1.2, we now find that

$$
\operatorname{diam}(X)>X^{1-1 / k-0.79155 / k}=X^{0.10423}
$$

if $X$ is sufficiently large (the constant -0.79155 plays the role of $-1 /(n-1)-$ $\varepsilon / 2$ in the proof).

More generally, we have 
Lemma 3.2. Suppose $n, k$ and $a_{i}$ are given as above, and suppose that we have a simultaneous irrationality measure of $\lambda$ for the following set of numbers: $\left\{\left(a_{i} / a_{n}\right)^{1 / k} \mid i=1, \ldots, n-1\right\}$. Then

$$
\operatorname{diam}(X)>X^{1-1 / k+(-\lambda+1) / k}=X^{1-\lambda / k},
$$

if $X$ is sufficiently large. Furthermore, this lower bound for the exponent of $X$ is trivial, resp. optimal precisely when $\lambda$ is.

P r o o f. Our example shows how to derive the first assertion. As concerns the second claim, we have $1=X^{0} \leq \operatorname{diam}(X)$ as a trivial lower bound; this corresponds to $\lambda=k$, which is the trivial upper bound for $\lambda$. On the other hand, the optimal exponent for $X$ is $1-1 / k-1 /(k(n-1))-\delta$. This corresponds to $\lambda=1+1 /(n-1)+k \delta$, which is optimal for $\lambda$ as well (cf. [3]).

For more examples, including higher values of both $n$ and $k$, we refer to Bennett's paper [3].

4. How to find examples of large or small diameter. In the sequel, we generate solutions to the Diophantine approximation systems on which the proofs of Theorems 1.1 and 1.3 are based, by finding short elements in suitable lattices (cf. [9] for an overview of this technique). The computational burden of such searches may be reduced by using a suitable form of lattice basis, e.g. an $L^{3}$-reduced basis. This has the additional notational benefit that the coefficients of the interesting lattice points with respect to the chosen basis will be small. However, we leave the actual implementation of the search to the number theory package KASH, which has a built-in feature, termed lattice enumeration, for finding all points $\boldsymbol{x}$ of a lattice such that

$$
0 \leq L \leq\left\|\boldsymbol{x}-\boldsymbol{x}_{\mathbf{0}}\right\| \leq R
$$

for a lower bound $L$, an upper bound $R$, and a reference vector $\boldsymbol{x}_{\mathbf{0}}$ (which is not necessarily a lattice point) $\left({ }^{1}\right)$.

We take again our standard example, $k=2, n=3, a_{1}=3, a_{2}=2$, $a_{3}=1$. Then, as noted above,

$$
\vartheta=\frac{2}{3} \sqrt{3}(1+\sqrt{2}) \approx 2.0975096, \quad \psi=2 \sqrt[4]{1}(\sqrt{3}+\sqrt{2}) \approx 6.2925287 .
$$

Let us first look for $X$ in the order of magnitude of $10^{12}$ with small $\operatorname{diam}(X)$. Proceeding as in the proof of Theorem 1.3, we look for $x_{1}, x_{2}, x_{3} \in$

$\left({ }^{1}\right)$ All software mentioned in this section may be obtained in digital form from the second author. The KASH Computer Algebra package is freely available from the ftp site of the Technische Universität Berlin (host ftp.math.tu-berlin.de, directory / pub/algebra/ Kant/Kash). More information may be obtained via WWW at http://www.math. tu-berlin.de/ ${ }^{\sim}$ kant/kash.html. 
$\mathbb{Z}$ which have the magnitude of $X^{1 / k}$, i.e. $10^{6}$, and with

$$
\left\{\begin{array}{l}
\left|x_{1}-x_{3} / \sqrt{3}\right| \approx 10^{-3} \\
\left|x_{2}-x_{3} / \sqrt{2}\right| \approx 10^{-3}
\end{array}\right.
$$

We consider the lattice

$$
\Gamma=\left\{\mathcal{A} \boldsymbol{x} \mid \boldsymbol{x} \in \mathbb{Z}^{3}\right\}
$$

where the matrix $\mathcal{A}$ is given by

$$
\mathcal{A}=\left[\begin{array}{ccc}
10^{9} & 0 & -\left[10^{9} / \sqrt{3}\right] \\
0 & 10^{9} & -\left[10^{9} / \sqrt{2}\right] \\
0 & 0 & 1
\end{array}\right]=\left[\begin{array}{ccc}
10^{9} & 0 & -577350269 \\
0 & 10^{9} & -707106781 \\
0 & 0 & 1
\end{array}\right]
$$

By Theorem 1.2, we expect that if we take $\boldsymbol{x}=\left(x_{1}, x_{2}, x_{3}\right)$ as indicated above, then the first or second component, or both, of the corresponding lattice point $\mathcal{A} \boldsymbol{x}$ should have at least the same magnitude. As the length of a vector is nearly proportional to its largest component, we can assume that all interesting triples correspond to lattice elements that are among the shortest in the lattice.

This approach turns out to be practical; in fact, if we calculate an $L^{3}$ reduced basis for $\Gamma$, one of the basis vectors corresponds to $x_{1}=565499$, $x_{2}=692592, x_{3}=979473$, and thus to $X=979473^{2}=959367357729$. Let us calculate its diameter: we have

$$
\begin{gathered}
3 x_{1}^{2}=959367357003<X, \quad 2 x_{2}^{2}=959367356928<3 x_{1}^{2}, \\
x_{3}^{2}=959367357729=X .
\end{gathered}
$$

Thus we have $\operatorname{diam}(X) \leq X-2 x_{2}^{2}=801$; one easily verifies that the other possibilities do not yield a smaller value for the diameter. Now 801 is small, since $\operatorname{diam}(X) / \sqrt[4]{X}=0.8093 \ldots$ is considerably less than $\psi$. Taking other linear combinations of these basis vectors we find lattice points leading to other interesting examples.

Next we look for $X$ in the order of magnitude of $10^{12}$ with large diam $(X)$; we follow the proofs of Corollary 2.4 and Proposition 2.3. It is easy to verify that the index subset $I$ such that $\frac{k}{|I|+1} \sum_{j \in I} a_{j}^{1 / k}$ is maximal is equal to $\{1,2\}$. Thus we have $T=\{1,2\}$ and $m=2$; however, we may take either $t_{1}=1$ and $t_{2}=2$ or $t_{1}=2$ and $t_{2}=1$. We choose the former possibility; it may be noted that reversing the order changes the values of the $\gamma_{s}$ that we are about to calculate, and thus yields other examples, as these are constructed from solutions of (10).

Solving the resulting linear system (9), we find $\gamma_{1}=\frac{2}{3}(\sqrt{3}+\sqrt{2})$, and $\gamma_{2}=\frac{2}{3}(2 \sqrt{2}-\sqrt{3})$. As $a_{1} \neq 1 \neq a_{2}$, we may take $b=1$. Hence we want 
$x_{1}, x_{2}, y \in \mathbb{Z}$ with

$$
\left\{\begin{array}{l}
\left|x_{1}-y / \sqrt{3}+\frac{\sqrt{3}+\sqrt{2}}{3 \sqrt{3}}\right| \approx 10^{-3}, \\
\left|x_{2}-y / \sqrt{2}+\frac{2 \sqrt{2}-\sqrt{3}}{3 \sqrt{2}}\right| \approx 10^{-3}
\end{array}\right.
$$

as $y^{-1 / 2} \approx\left(10^{6}\right)^{-1 / 2}$. Thus it turns out that we can use the same lattice as above, but now look for lattice points in the neighborhood of the (nonlattice) point

$$
\boldsymbol{y}=\left(\begin{array}{c}
-\left[10^{9} \frac{\sqrt{3}+\sqrt{2}}{3 \sqrt{3}}\right] \\
-\left[10^{9} \frac{2 \sqrt{2}-\sqrt{3}}{3 \sqrt{2}}\right] \\
0
\end{array}\right)=\left(\begin{array}{c}
-605498860 \\
-258418376 \\
0
\end{array}\right)
$$

According to KASH, the lattice point nearest to $\boldsymbol{y}$ is

$$
\boldsymbol{b}_{\mathbf{0}}=\left(\begin{array}{c}
-605051719 \\
-258857831 \\
-147949
\end{array}\right)=\mathcal{A}\left(\begin{array}{c}
-85419 \\
-104616 \\
-147949
\end{array}\right) \text {. }
$$

Thus $x_{1}=-85419, x_{2}=-104616, y=-147949$ is of interest: taking $X=$ $147949^{2}$, we find $\operatorname{diam}(X)=310082$, which is large, since $310082 / 147949=$ $2.0958 \ldots$ is almost $\vartheta$. The signs of the values do not matter, as $k$ is even.

We can increase diam $(X)$ even more by taking an $X$ of general form, as indicated in the proof of Theorem 1.2. In this case, $X-34$ turns out to be the real local maximum of the diam-function, with $\operatorname{diam}(X-34)=310116$.

Again, looking at other lattice points in the neighborhood produces more interesting examples.

Extending these computations to larger values of $n$ and $k$ is not difficult. For example, we have employed our algorithm to find all $X$ between $3 \cdot 10^{3}$ and $3 \cdot 10^{31}$ with $\operatorname{diam}(X) \leq 4 \cdot X^{5 / 9}$, taking $n=4, k=3, a_{1}=7, a_{2}=5$, $a_{3}=3, a_{4}=2$. The "best" example we thus found has got

$$
\begin{array}{ll}
x_{1}=53240, & 7 x_{1}^{3}=1056360565568000, \\
x_{2}=59559, & 5 x_{2}^{3}=1056360604069395, \\
x_{3}=70615, & 3 x_{3}^{3}=1056360479575125, \\
x_{4}=80834, & 2 x_{4}^{3}=1056360627067408 .
\end{array}
$$

Locating the local maximum, we found

$$
X=1056360627067408, \quad \operatorname{diam}(X)=147492283,
$$

giving a ratio $\operatorname{diam}(X) / X^{5 / 9}$ of $0.664 \ldots$ This search took KASH less than one minute on an SGI Indy workstation with an R5000 processor. 


\section{References}

[1] A. Baker, The theory of linear forms in logarithms, in: A. Baker and D. W. Masser (eds.), Transcendence Theory: Advances and Applications, London, 1977, 1-27.

[2] A. Baker and H. Davenport, The equations $3 x^{2}-2=y^{2}$ and $8 x^{2}-7=z^{2}$, Quart. J. Math. Oxford Ser. (2) 20 (1969), 129-137.

[3] M. A. Bennett, Simultaneous rational approximation to binomial functions, Trans. Amer. Math. Soc. 348 (1996), 1717-1738.

[4] G. V. Chudnovsky, On the method of Thue-Siegel, Ann. of Math. (2) 117 (1983), 325-382.

[5] W. M. Schmidt, Diophantine Approximation, Lecture Notes in Math. 785, Springer, Berlin, 1980.

[6] M. M. Sweet, A theorem in Diophantine approximations, J. Number Theory 5 (1973), 245-251.

[7] J. Turk, Almost powers in short intervals, Arch. Math. (Basel) 43 (1984), 157-166.

[8] N. Tzanakis and B. M. M. de Weger, On the practical solution of the Thue equation, J. Number Theory 31 (1989), 99-132.

[9] B. M. M. de Weger, Algorithms for Diophantine Equations, CWI Tract 65, Centrum Wisk. Inform., Amsterdam, 1989, 19-26.

[10] B. M. M. de Weger and C. E. van de Woestijne, On the power-free parts of consecutive integers, Acta Arith., this issue, 387-395.

Sportsingel 30

2924 XN Krimpen aan den IJssel

The Netherlands

E-mail: deweger@xs4all.nl
Mathematical Institute

Universiteit Leiden

P.O. Box 9512, 2300 RA Leiden

The Netherlands

E-mail: cvdwoest@wi.LeidenUniv.nl

Received on 23.10.1998

and in revised form on 30.4.1999 